\title{
Constraints on the quark matter equation of state from astrophysical observations
}

\section{G. F. Burgio*}

INFN Sezione di Catania, via S. Sofia 64, I-95123 Catania, Italy

E-mail: fiorella.burgiodet.infn.it

\section{H. Chen}

Physics Department, China University of Geoscience, Wuhan 430074, China

E-mail: huanchen.phydgmail.com

\section{H.-J. Schulze}

INFN Sezione di Catania, via S. Sofia 64, I-95123 Catania, Italy

E-mail: schulzedct.infn.it

\section{G. Taranto}

Dipartimento di Fisica e Astronomia, Universita' di Catania, and INFN Sezione di Catania, via

S. Sofia 64, I-95123 Catania, Italy

E-mail: tarantogabrieledgmail.com

We calculate the structure of neutron star interiors comprising both the hadronic and the quark phases. For the hadronic sector we employ a microscopic equation of state involving nucleons and hyperons derived within the Brueckner-Hartree-Fock many-body theory with realistic two-body and three-body forces. For the description of quark matter, we use several different models, e.g. the MIT bag, the Nambu-Jona-Lasinio (NJL), the Color Dielectric (CDM), the Field Correlator method (FCM), and one based on the Dyson-Schwinger model (DSM). We find that a two solar mass hybrid star is possible only if the nucleonic EOS is stiff enough.

Xth Quark Confinement and the Hadron Spectrum,

October 8-12, 2012

TUM Campus Garching, Munich, Germany

\footnotetext{
${ }^{*}$ Speaker.
} 
The possible appearance of quark matter (QM) in the interior of massive neutron stars (NS) is one of the main issues in the physics of these compact objects. Calculations of NS structure, based on a microscopic nucleonic equation of state (EOS), indicate that for the heaviest NS, close to the maximum mass (about two solar masses), the central particle density reaches values larger than $1 / \mathrm{fm}^{3}$. In this density range the nucleons start to loose their identity, and quark degrees of freedom play a role.

The value of the maximum mass of a NS is probably one of the physical quantities that are most sensitive to the presence of QM, and the recent claim of discovery of a two solar mass NS [四] has stimulated the interest in this issue. Unfortunately, while the microscopic theory of the nucleonic EOS has reached a high degree of sophistication [ [2], the QM EOS is poorly known at zero temperature and at the high baryonic density appropriate for NS. One has, therefore, to rely on models of QM, which contain a high degree of arbitrariness. At present, the best one can do is to compare the predictions of different quark models and to estimate the uncertainty of the results for the NS matter as well as for the NS structure and mass.

In this paper we will discuss a set of different quark models in combination with a definite baryonic EOS, which has been developed within the Brueckner-Hartree-Fock (BHF) many-body

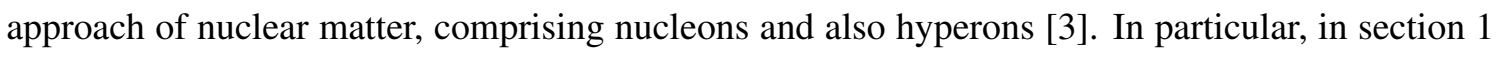
we review the derivation of the baryonic EOS in the BHF approach, whereas Section $\square$ is devoted to the relevant features of the hadron quark phase transition for the several QM EOS discussed. In section [] we present the results regarding NS structure, combining the baryonic and QM EOS for beta-stable nuclear matter, and conclusions are drawn.

\section{EOS of nuclear matter within Brueckner theory}

The EOS constructed for the hadronic phase at $T=0$ is based on the non-relativistic BruecknerBethe-Goldstone (BBG) many-body theory [3], which is a linked cluster expansion of the energy per nucleon of nuclear matter, well convergent and accurate enough in the density range relevant for neutron stars. In this approach the essential ingredient is the two-body scattering matrix $G$, which, along with the single-particle potential $U$, satisfies the self-consistent equations

$$
\begin{aligned}
G(\rho ; \omega) & =V+V \sum_{k_{a} k_{b}} \frac{\left|k_{a} k_{b}\right\rangle Q\left\langle k_{a} k_{b}\right|}{\omega-e\left(k_{a}\right)-e\left(k_{b}\right)} G(\rho ; \omega), \\
U(k ; \rho) & =\sum_{k^{\prime} \leq k_{F}}\left\langle k k^{\prime}\left|G\left(\rho ; e(k)+e\left(k^{\prime}\right)\right)\right| k k^{\prime}\right\rangle_{a},
\end{aligned}
$$

where $V$ is the bare nucleon-nucleon (NN) interaction, $\rho$ is the nucleon number density, $\omega$ is the starting energy, $\left|k_{a} k_{b}\right\rangle Q\left\langle k_{a} k_{b}\right|$ is the Pauli operator, $e(k)=e(k ; \rho)=\frac{\hbar^{2}}{2 m} k^{2}+U(k ; \rho)$ is the single particle energy, and the subscript " $a$ " indicates antisymmetrization of the matrix element. In the BHF approximation the energy per nucleon is

$$
\begin{aligned}
& \frac{E}{A}(\rho)=\frac{3}{5} \frac{\hbar^{2} k_{F}^{2}}{2 m}+D_{2}, \\
& D_{2}=\frac{1}{2 A} \sum_{k, k^{\prime} \leq k_{F}}\left\langle k k^{\prime}\left|G\left(\rho ; e(k)+e\left(k^{\prime}\right)\right)\right| k k^{\prime}\right\rangle_{a} .
\end{aligned}
$$


The inclusion of nuclear three-body forces (TBF) is crucial in order to reproduce the correct saturation point of symmetric nuclear matter [ [ 9 , []]. The present theoretical status of microscopically derived TBF is quite rudimentary. Recent results [ $[\mathbf{6}, \mathbf{\square}]$ have shown that both two-body and threebody forces should be based on the same theoretical footing and use the same microscopical parameters in their construction. Results shown here were obtained with the Argonne $V_{18}$ (V18) [8] or the Bonn B (BOB) [Q] potentials, and compared also with the widely used phenomenological Urbana-type (UIX) TBF [ए]] (in combination with the V18 potential). It should be stressed that in our approach the TBF is reduced to a density-dependent two-body force by averaging over the position of the third particle, assuming that the probability of having two particles at a given distance is given by the two-body correlation function determined self-consistently.

In the past years, the BHF approach has been extended with the inclusion of hyperons [ए], प2, [13], which may appear at baryon density of about 2 to 3 times normal nuclear matter density. The inclusion of hyperons produces an EOS which turns out to be much softer than the purely nucleonic case, with dramatic consequences for the structure of the NS, i.e., the value of the maximum mass is smaller than the canonical value $1.44 M_{\odot}$. The inclusion of further theoretical ingredients, such as hyperon-hyperon potentials [ㅁ] ] and/or three-body forces involving hyperons, could alter the baryonic EOS, but unfortunately they are essentially unknown. Another possibility that is able to produce larger maximum masses, is the appearance of a transition to QM inside the star. This scenario will be illustrated below.

Starting from the EOS for symmetric and pure neutron matter, and assuming stellar matter composed of neutrons, protons, and leptons [四], the EOS for the beta equilibrated matter can be obtained in the usual standard way [4, [4, [5]]: The Brueckner calculation yields the energy density of baryon/lepton matter as a function of the different partial densities, $\varepsilon\left(\rho_{n}, \rho_{p}, \rho_{e}, \rho_{\mu}\right)$. The various chemical potentials for the species $i=n, p, e, \mu$ can then be computed straightforwardly,

$$
\mu_{i}=\frac{\partial \varepsilon}{\partial \rho_{i}}
$$

and the equations for beta-equilibrium,

$$
\mu_{i}=b_{i} \mu_{n}-q_{i} \mu_{e}
$$

( $b_{i}$ and $q_{i}$ denoting baryon number and charge of species $i$ ) and charge neutrality,

$$
\sum_{i} \rho_{i} q_{i}=0
$$

allow one to determine the equilibrium composition $\left\{\rho_{i}(\rho)\right\}$ at given baryon density $\rho$ and finally the EOS,

$$
P(\rho)=\rho^{2} \frac{d}{d \rho} \frac{\varepsilon\left(\left\{\rho_{i}(\rho)\right\}\right)}{\rho}=\rho \frac{d \varepsilon}{d \rho}-\varepsilon=\rho \mu_{n}-\varepsilon .
$$

In Fig. Wwe compare the EOS obtained in the BHF framework when only nucleons and leptons are present (thick lines), and the corresponding ones with hyperons included (thin lines). Calculations have been performed with different choices of the NN potentials, i.e., the Bonn B, the Argonne V18, and the Nijmegen N93, all supplemented by a compatible microscopic TBF [ 6 ]. For completeness, 


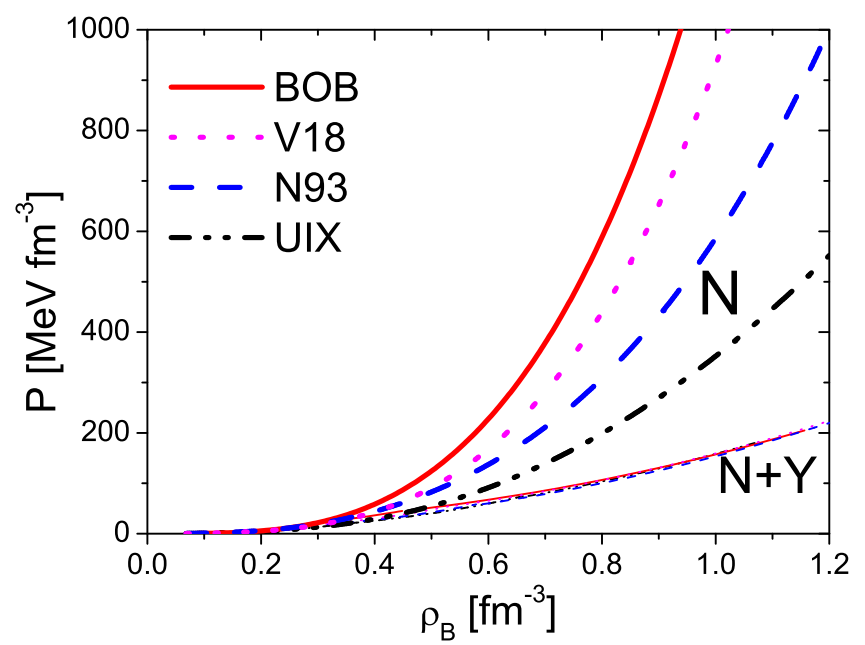

Figure 1: Pressure vs. the baryon number density of hadronic NS matter. Thick curves show results for purely nucleonic matter, whereas thin curves include hyperons.

we also show results obtained with the Argonne V18 potential together with the phenomenological Urbana IX as TBF.

We notice a strong dependence on both the chosen NN potential, and on the adopted TBF, the microscopic one being more repulsive than the phenomenological force. The presence of hyperons decreases strongly the pressure, and the resulting EOS turns out to be almost independent of the adopted NN potential, due to the interplay between the stiffness of the nucleonic EOS and the threshold density of hyperons [ [6]. The softening of the EOS has serious consequences for the structure of NS, leading to a maximum mass of less than 1.4 solar masses [ [0, [3] ], which is below the observed pulsar masses [ए]].

\section{Quark Phase}

The properties of cold nuclear matter at large densities, i.e., its EOS and the location of the phase transition to deconfined QM, remain poorly known. The difficulty in performing firstprinciple calculations in such systems can be traced back to the complicated nonlinear and nonperturbative nature of quantum chromodynamics (QCD). Therefore one can presently only resort to more or less phenomenological models for describing QM.

One of the most widely used approach is the MIT bag model [1]7]. In the calculations shown below, we assumed massless $u$ and $d$ quarks, $s$ quarks with a current mass of $m_{s}=150 \mathrm{MeV}$, and either a fixed bag constant $B=90 \mathrm{MeV} \mathrm{fm}^{-3}$, or a density-dependent bag parameter,

$$
B(\rho)=B_{\infty}+\left(B_{0}-B_{\infty}\right) \exp \left[-\beta\left(\frac{\rho}{\rho_{0}}\right)^{2}\right]
$$

with $B_{\infty}=50 \mathrm{MeVfm}^{-3}, B_{0}=400 \mathrm{MeVfm}^{-3}$, and $\beta=0.17$. This approach has been proposed in [ए8], and it allows the symmetric nuclear matter to be in the pure hadronic phase at low densities, and in the quark phase at large densities, while the transition density is taken as a parameter. We 

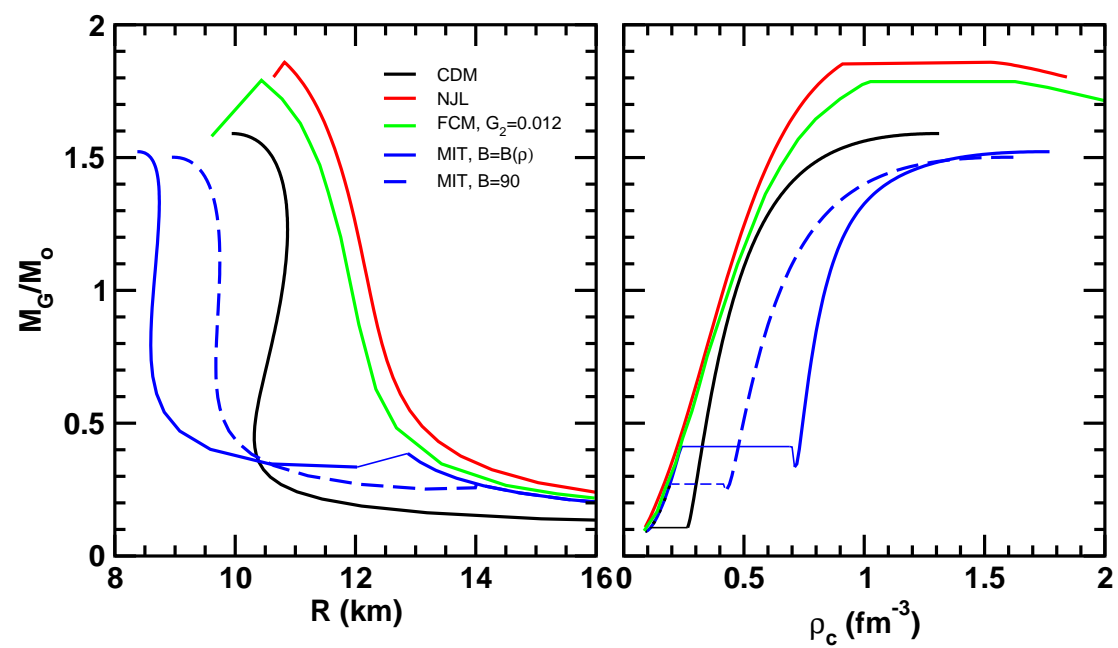

Figure 2: Gravitational NS mass vs. the radius (left panel) and the central baryon density (right panel) for different EOS of quark matter. See text for details.

find that the phase transition takes place at baryon density of the order of $2-3 \rho_{0}$, and that the mixed phase contains a small fraction of hyperons.

The phase transition from the hadronic phase to the pure quark matter phase is usually performed through the Maxwell construction, or the more sophisticated Gibbs construction [U5]. This is widely used for the study of neutron star structure, several numerical details can be found in many references [ㄷ]], and will not be repeated here. The important point to stress is that the features of the phase transition, such as its onset and the density interval over which it extends, depend crucially on the models used for describing the hadron and the quark phase. For example, we found that the phase transition constructed with the CDM [ㅁ] model is quite different from the one obtained using the MIT bag model. In the CDM, the onset of the coexistence region occurs at very low baryonic density. This implies a large difference in the structure of neutron stars. In fact, whereas stars built with the CDM have at most a mixed phase at low density and a pure quark core at higher density, the ones obtained with the MIT bag model contain a hadron phase, followed by a mixed phase and a pure quark interior. The scenario is again different within the NambuJona-Lasinio model [[2]], where at most a mixed phase is present, but no pure quark phase. It has also been found that the phase transition from hadronic to QM occurs at high values of the baryon chemical potential when the Dyson-Schwinger model is used to describe the quark phase. In some extreme cases, for particular choices of the parameters, no phase transition at all is possible. In fact, the Dyson-Schwinger EOS [2]] is generally stiffer than the hadronic one, and the value of the transition density is high. We also found that with the DSM no phase transition exists if the hadronic phase contains hyperons, just like the phase transition with NJL model.

It is worthwhile to mention that neutron stars observations can help to determine free parameters, which could be present in some quark matter models. This is the case of the QM EOS based on the Field Correlator method [22], which depends crucially on the value of the gluon condensate $G_{2}$. It turns out that using value of $G_{2} \simeq 0.006-0.007 \mathrm{GeV}^{4}$, which gives a critical temperature $\mathrm{T}_{\mathrm{c}} \simeq 170 \mathrm{MeV}$, produces maximum masses which are only marginally consistent with the obser- 
vational limit, while larger masses are possible if the value of the gluon condensate is increased. Also in this case, the phase transition only takes place if no hyperons are present in the hadronic phase.

\section{Neutron star structure}

We assume that a NS is a spherically symmetric distribution of mass in hydrostatic equilibrium. The equilibrium configurations are obtained by solving the Tolman-Oppenheimer-Volkoff (TOV) equations [14] for the pressure $P$ and the enclosed mass $m$,

$$
\begin{aligned}
& \frac{d P}{d r}=-\frac{G m \varepsilon}{r^{2}} \frac{(1+P / \varepsilon)\left(1+4 \pi r^{3} P / m\right)}{1-2 G m / r} \\
& \frac{d m}{d r}=4 \pi r^{2} \varepsilon
\end{aligned}
$$

being $G$ the gravitational constant. Starting with a central mass density $\varepsilon(r=0) \equiv \varepsilon_{c}$, we integrate out until the density on the surface equals the one of iron. This gives the stellar radius $R$ and the gravitational mass is then

$$
M_{G} \equiv m(R)=4 \pi \int_{0}^{R} d r r^{2} \varepsilon(r) .
$$

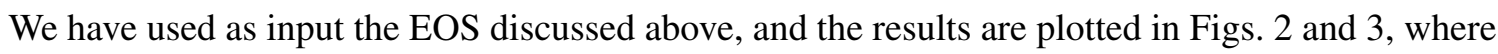
we display the gravitational mass $M_{G}$ (in units of the solar mass $M_{\odot}=2 \times 10^{33} \mathrm{~g}$ ) as a function of the radius $R$ and central baryon density $\rho_{c}$.

Calculations displayed in Fig. $\square$ for neutron star matter are obtained in the BHF theoretical framework with the V18 nucleon-nucleon potential in combination with i) the CDM (solid black curve), and ii) the MIT bag model (blue curves) for quark matter. Due to the use of the Maxwell construction, the curves are not continuous [ㄷ]]: for very small central densities (large radii, small masses) the stars are purely hadronic. We observe that the values of the maximum mass depend only slightly on the EOS chosen for describing quark matter, and lie between 1.5 and 1.6 solar masses. A clear difference between the two models exists as far as the radius is concerned. Hybrid stars built with the CDM are characterized by a larger radius and a smaller central density, whereas hybrid stars constructed with the MIT bag model are more compact. Further calculations have been performed using the Paris potential as nucleon-nucleon interaction for the hadronic phase, and the NJL model for the quark phase (red curve). In this case the onset of pure quark matter leads to an instability, as well as in the FCM (green curve). In both cases the phase transition takes place only if no hyperons are present in the hadronic phase. Unfortunately, for all the cases discussed above, the value of the maximum mass lies below the mass observed for the pulsar PSR J1614-2230, $M_{\odot}=1.97 \pm 0.04$ [四]. Such a high value puts severe constraints on the EOS, and in particular it demands an additional repulsion in the QM EOS.

In Fig. B we show results obtained in the BHF framework using the Bonn B nucleon-nucleon potential, which produces the stiffest EOS, as shown in Fig. 1 . This yields a very high maximum NS mass, $\approx 2.50 M_{\odot}$, with only nucleons (solid black curve), and $1.37 M_{\odot}$ including hyperons. Using the DSM for the quark phase, we found that the maximum mass of hybrid stars is only a little lower than $2.5 M_{\odot}$ with $\alpha=0.5$, and decreases to about $2 M_{\odot}$ with $\alpha=2$, being $\alpha$ a 


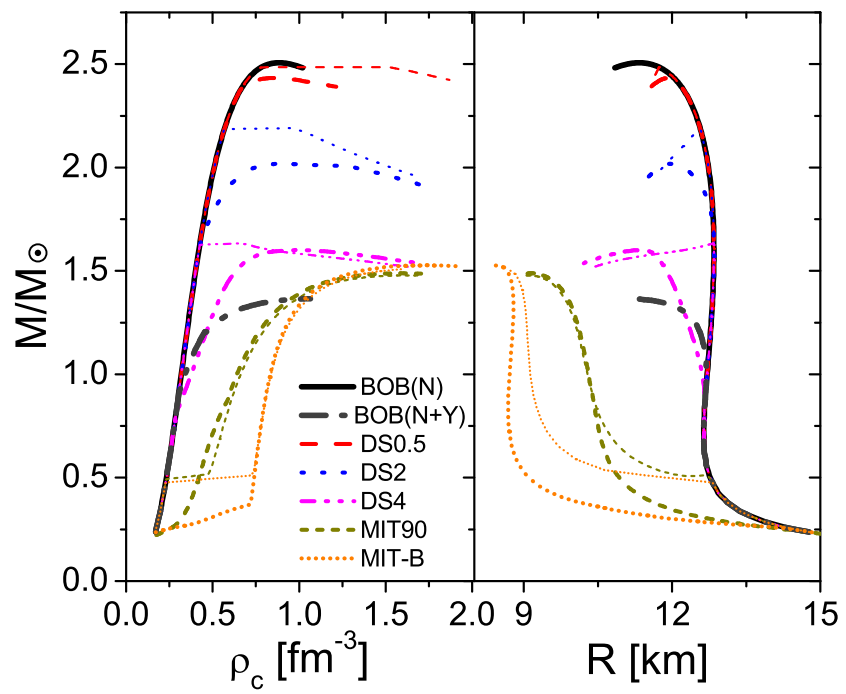

Figure 3: Gravitational NS mass vs. the radius (right panel) and the central baryon density (left panel) for different EOS employing the BOB hadronic model.

model parameter which controls the rate of approaching asymptotic freedom. With increasing $\alpha$ we can obtain a smooth change from the pure hadronic NS to the results with the MIT bag model. Moreover, no phase transition can occur and no hybrid star can exist if hyperons are introduced. If hyperons are excluded, the phase transition from nucleon matter to QM takes place at rather large baryon density, and the onset of the phase transition is determined in this case by the parameter $\alpha$.

The possible effects of the hadron-quark phase transition are very different with the MIT bag model and the DSM: In the case of the MIT model, the phase transition begins at very low baryon density and thus effectively impedes the appearance of hyperons. Consequently the resulting maximum mass of the MIT hybrid star is $1.5 M_{\odot}$, lower than the value of the nucleonic star, but higher than that of the hyperon star given before. A clear difference between the two models exists as far as the radius is concerned. Hybrid stars built with the DSM are characterized by a larger radius and a smaller central density, whereas hybrid stars constructed with the MIT bag model are more compact.

In conclusion, a hybrid star with $2 M_{\odot}$ is only allowed if the nucleonic EOS is stiff enough, and the hadron-quark phase transition takes place without hyperons in the hadronic phase.

\section{References}

[1] P. B. Demorest, T. Pennucci, S. M. Ransom, M. S. E. Roberts, and J. W. T. Hessels, Nature 467, 1081 (2010).

[2] M. Baldo and G. F. Burgio, Rep. Prog. Phys. 75, 026301 (2012).

[3] M. Baldo, Nuclear Methods and the Nuclear Equation of State, International Review of Nuclear Physics, Vol. 8 (World Scientific, Singapore, 1999).

[4] M. Baldo, I. Bombaci, and G. F. Burgio, Astron. Astrophys. 328, 274 (1997). 
[5] A. Akmal, V. R. Pandharipande, and D. G. Ravenhall, Phys. Rev. C58, 1804 (1998).

[6] Z. H. Li and H.-J. Schulze, Phys. Rev. C78, 028801 (2008).

[7] A. Lejeune, P. Grangé, M. Martzolff, and J. Cugnon, Nucl. Phys. A453, 189 (1986); W. Zuo, A. Lejeune, U. Lombardo, and J.-F. Mathiot, Nucl. Phys. A706, 418 (2002); Z. H. Li, U. Lombardo, H.-J. Schulze, and W. Zuo, Phys. Rev. C77, 034316 (2008).

[8] R. B. Wiringa, V. G. J. Stoks, and R. Schiavilla, Phys. Rev. C51, 38 (1995).

[9] R. Machleidt, K. Holinde, and Ch. Elster, Phys. Rep. 149, 1 (1987); R. Machleidt, Adv. Nucl. Phys. 19, 189 (1989).

[10] J. Carlson, V. R. Pandharipande, and R. B. Wiringa, Nucl. Phys. A401, 59 (1983); R. Schiavilla, V. R. Pandharipande, and R. B. Wiringa, Nucl. Phys. A449, 219 (1986); B. S. Pudliner, V. R. Pandharipande, J. Carlson, S. C. Pieper, and R. B. Wiringa, Phys. Rev. C56, 1720 (1997).

[11] H.-J. Schulze, M. Baldo, U. Lombardo, J. Cugnon, and A. Lejeune, Phys. Rev. C57, 704 (1998); M. Baldo, G. F. Burgio, and H.-J. Schulze, Phys. Rev. C58, 3688 (1998); Phys. Rev. C61, 055801 (2000).

[12] I. Vidaña, A. Polls, A. Ramos, M. Hjorth-Jensen, and V. G. J. Stoks, Phys. Rev. C61, 025802 (2000).

[13] H.-J. Schulze, A. Polls, A. Ramos, and I. Vidaña, Phys. Rev. C73, 058801 (2006).

[14] S. L. Shapiro and S. A. Teukolsky, Black Holes, White Dwarfs, and Neutron Stars (John Wiley \& Sons, New York, 1983).

[15] N. K. Glendenning, Compact Stars, Nuclear Physics, Particle Physics, and General Relativity, 2nd ed., 2000, Springer-Verlag, New York.

[16] J. M. Lattimer and M. Prakash, Phys. Rep. 442, 109 (2007).

[17] A. Chodos, R. L. Jaffe, K. Johnson, C. B. Thorn, and V. F. Weisskopf, Phys. Rev. D9, 3471 (1974).

[18] G. F. Burgio, M. Baldo, P. K. Sahu, and H.-J. Schulze, Phys. Rev. C66, 025802 (2002).

[19] C. Maieron, M. Baldo, G. F. Burgio, and H.-J. Schulze, Phys. Rev. D70, 043010 (2004).

[20] M. Baldo, M. Buballa, G. F. Burgio, F. Neumann, M. Oertel, and H.-J. Schulze, Phys. Lett. B562, 153 (2003); M. Baldo, G. F. Burgio, P. Castorina, S. Plumari, and D. Zappalà, Phys. Rev. C75, 035804 (2007).

[21] H. Chen, M. Baldo, G. F. Burgio, and H.-J. Schulze, Phys. Rev. D84, 105023 (2011).

[22] M. Baldo, G. F. Burgio, P. Castorina, S. Plumari, and D. Zappalà, Phys. Rev. D78, 063009 (2008). 